\title{
Defining the Normal Trends of Serum Creatine Kinase Levels Following Spinal Surgery
}

\author{
Matthew Griffith, Kenneth Aaron Shaw, Michael Baird, \\ Patrick Rushford, Victoria Shaw, Aaron Roberts, David M. Gloystein \\ Department of Orthopaedic Surgery, Dwight David Eisenhower Army Medical Center, Fort Gordon, GA, USA
}

\begin{abstract}
Study Design: Prospective, prognostic study, level II evidence.
Purpose: To define the normal change in the creatine kinase (CK) levels in patients undergoing prone or supine lumbar or cervical spine surgery and to determine if positioning influences the postoperative changes in the CK levels.

Overview of Literature: Spine surgery is one of the most commonly performed and fastest growing areas of surgery in the United States. Thus, the various possible complications need to be understood, and risk factors for these complications need to be mitigated. One of the rare complications, reported in the literature as small case series and case reports, is rhabdomyolysis, diagnosed by high CK levels. Thus far, very few studies have examined the rise in CK levels following spine surgery, and to our knowledge, none has assessed the potential association of surgical positioning and the rise in CK levels.

Methods: We retrospectively analyzed 94 patients. We obtained their preoperative CK levels, and re-assessed their CK levels at postoperative day (POD) 1, 2, and 3, as well as at their 2-week follow-up. The data were analyzed with respect to the spine level and positioning to determine if positioning had any effect on the postoperative rise in the CK level.

Results: Total 94 consecutive patients were enrolled in this study. The average preoperative CK level was 179.64, and the average CK level was 847.04 on POD 1. Prone positioning showed a greater rise in the CK levels following surgery than the supine positioning. In a similar manner, lumbar procedures led to a larger rise in the CK levels than cervical surgery. Prone/lumbar surgery showed the largest increase among all groups. Finally, revision surgery and instrumentation both increased the postoperative CK levels.

Conclusions: This study demonstrated that positioning can affect the postoperative CK level rise, with patients undergoing prone/ lumbar surgery showing the greatest rise in the postoperative CK levels. This rise, however, may be related to paraspinal muscle damage, rather than the positioning itself.
\end{abstract}

Keywords: Spine surgery; Creatine kinase; Rhabdomyolysis

\footnotetext{
Received Jul 29, 2018; Revised Sep 7, 2018; Accepted Sep 12, 2018

Corresponding author: Matthew Griffith

Department of Orthopaedic Surgery, Dwight David Eisenhower Army Medical Center, 300 East Hospital Road, Fort Gordon, GA 30905, USA

Tel: +1-706-787-1859, Fax: +1-706-787-2565, E-mail: msg5041@gmail.com

*The opinions or assertions contained herein are the private views of the authors and are not to be construed as official or reflecting the views of the Department of Defense or US Government. The authors are employees of the US government. This work was prepared as part of their official duties and, as such there is no copyright to be transferred.
} 


\section{Introduction}

Spine surgery is one of the most common and fastest growing operating room procedures, with a rise from 485,000 laminectomies performed in 2001 to 488,000 performed in 2011, and 288,000 fusions in 2001 rising to 488,000 in 2011 [1]. Thus, it is important for surgeons to be familiar with the several possible complications, irrespective of their rarity. Rhabdomyolysis is one such rare but potentially devastating complication that involves the risk of acute kidney injury (AKI). AKI is reported to occur in $13 \%-50 \%$ of all rhabdomyolysis cases and is associated with a mortality rate of $20 \%-50 \%$ [2]. Several case reports/series have reported rhabdomyolysis and subsequent AKI after spinal procedures. Dakwar et al. [3] reported five cases of rhabdomyolysis and AKI in 315 patients undergoing spine surgery using the minimally invasive lateral approach. Creatine kinase (CK) levels of these patients ranged from 5,000-56,000 U/L with a mean of $25,861 \mathrm{U} / \mathrm{L}$ [3]. Other case studies show similar findings during lumbar spine fusion surgery [4].

Few studies have examined the change in the CK levels following surgery [5-8]. These studies, however, have either focused only on posterior lumbar surgery $[5,8]$, the degree of invasiveness [6], or have used a small sample size in a wide variety of procedures for a large number of diagnoses [7]. The present study aimed to determine the normal range of CK levels following elective spine procedures in active and dependent patients; we further aimed to determine whether the approach method influenced the development of rhabdomyolysis. Furthermore, this study was designed to assess whether positioning had any effect on the change in the serum CK values. This may assist spine surgeons in identifying patients at a higher risk of these rare and serious complications to enable more efficient management. We hypothesized that patients undergoing prone surgeries would have a larger rise in their postoperative $\mathrm{CK}$ values than those undergoing anterior procedures. We also speculated that a larger increase may be seen in patients undergoing prone surgery owing to a combination of pressure points over the muscular compartments with prone surgery as well as the increased muscle damage resulting from the dissection in posterior surgeries.

\section{Materials and Methods}

\section{Study design}

After obtaining approval from the Institutional Review Board of Dwight David Eisenhower Army Medical Center (IRB approval no., 404358), a prospective cohort study was initiated for patients undergoing elective spine surgery performed by a single fellowship-trained orthopedic spine surgeon at a single institution from January 2014 to December 2014.

\section{Patient population}

All the patients undergoing elective cervical or lumbar spine surgery were considered for participation. As per the inclusion criteria, adult patients aged $\geq 18$ years who were scheduled to undergo elective primary or revision cervical and lumbar spine surgery in either the prone or supine position were included. Exclusionary criteria included the presence of traumatic injuries, age $<18$ years, surgical intervention for an underlying infection or tumor, pregnancy, or a personal history of rhabdomyolysis. A preoperative power analysis determined the need for at least 35 patients in the supine and prone groups to capture the small effect sizes that were being studied. The study enrolled consecutive patients; therefore, a sample size of 150 was set to ensure at least 35 patients in each group, after excluding the patient dropouts.

\section{Data collection}

All the patients indicated for a surgical procedure were offered study participation, and those who agreed to participate were requested to provide informed consent during their normal, scheduled preoperative appointment. At the standard preoperative appointment, the CK level (units/ liter $[\mathrm{U} / \mathrm{L}])$ was assessed along with the patients standard preoperative labs, 1-14 days prior to the surgical intervention. Following surgery, all the patients underwent postoperative admission, with daily $\mathrm{CK}$ values being measured throughout the course of hospitalization.

In addition to these CK values, demographic information of the patients (age, sex, and ethnicity), preoperative renal function, intraoperative positioning, intraoperative blood loss, intraoperative fluid administration, number of levels operated on (defined as the number of traversed 
disc spaces), and surgical duration were recorded. The primary variable was the clinical diagnosis of rhabdomyolysis during the postoperative period. The study endpoint was the 2-week follow-up.

\section{Statistical analyses}

Statistical analyses were performed using SAS ver. 9.4 distribution (SAS Institute Inc., Cary, NC, USA), and the characteristics of all the variables were assessed. A between-group $t$-test was used to determine differences in the CK levels for surgery in the prone versus the supine position. Regression analysis was used to assess the preoperative or intraoperative factors predictive of elevated $\mathrm{CK}$ values. Prior to the statistical analyses, the data were screened for outliers, and the distributions were examined for skewness and kurtosis.

A correlation analysis, using Spearman's correlation coefficient, was performed to examine the relationship between preoperative $\mathrm{CK}$ and postoperative day (POD) $1 \mathrm{CK}, \mathrm{CK}$ change (difference) at POD 1 , and CK change (ratio) at POD 1 (POD 1/preoperative). Descriptive statistics were calculated for all the quantitative and qualitative variables. Wilcoxon rank-sum test was used for perform-

Table 1. Descriptive data of the patients undergoing surgery (all patients, $\mathrm{N}=94$ )

\begin{tabular}{lc} 
Variable & No. $(\%)$ \\
Sex & \\
Male & $66(70.21)$ \\
\hline Female & $28(29.79)$ \\
Race & \\
\hline White & $52(55.32)$ \\
\hline Black & $32(34.04)$ \\
\hline Other & $10(10.64)$ \\
No. of levels & \\
\hline 1 & $61(64.89)$ \\
\hline 2 & $21(22.34)$ \\
\hline$\geq 3$ & $12(12.77)$ \\
\hline Instrumentation & \\
\hline Yes & $80(85.11)$ \\
\hline No & $14(14.89)$ \\
\hline Revision & \\
\hline Yes & $6(6.38)$ \\
\hline No & $88(93.62)$ \\
\hline
\end{tabular}

ing comparisons between the two spinal regions (cervical versus lumbar), the two positions (prone versus supine), as well as instrumentation (yes/no) and revision (yes/ no). The Kruskal-Wallis test was used to compare the outcomes among the three groups defined by the number of levels (1,2, and 3 or more) and the four groups defined by the spinal region and the position (cervical/prone, cervi$\mathrm{cal} /$ supine, lumbar/prone, and lumbar/supine). An alpha level of 0.05 was used to indicate the statistical significance.

\section{Results}

Over the study period, 166 patients underwent spine surgery. Of these, 20 met the exclusion criteria, with all of them undergoing surgery for an underlying infection. Seventeen patients declined study participation. Of the remaining 134 patients, 19 failed to undergo preoperative CK measurement, and 21 had incomplete follow-up CK data sets, leaving a total of 94 patients for study inclusion

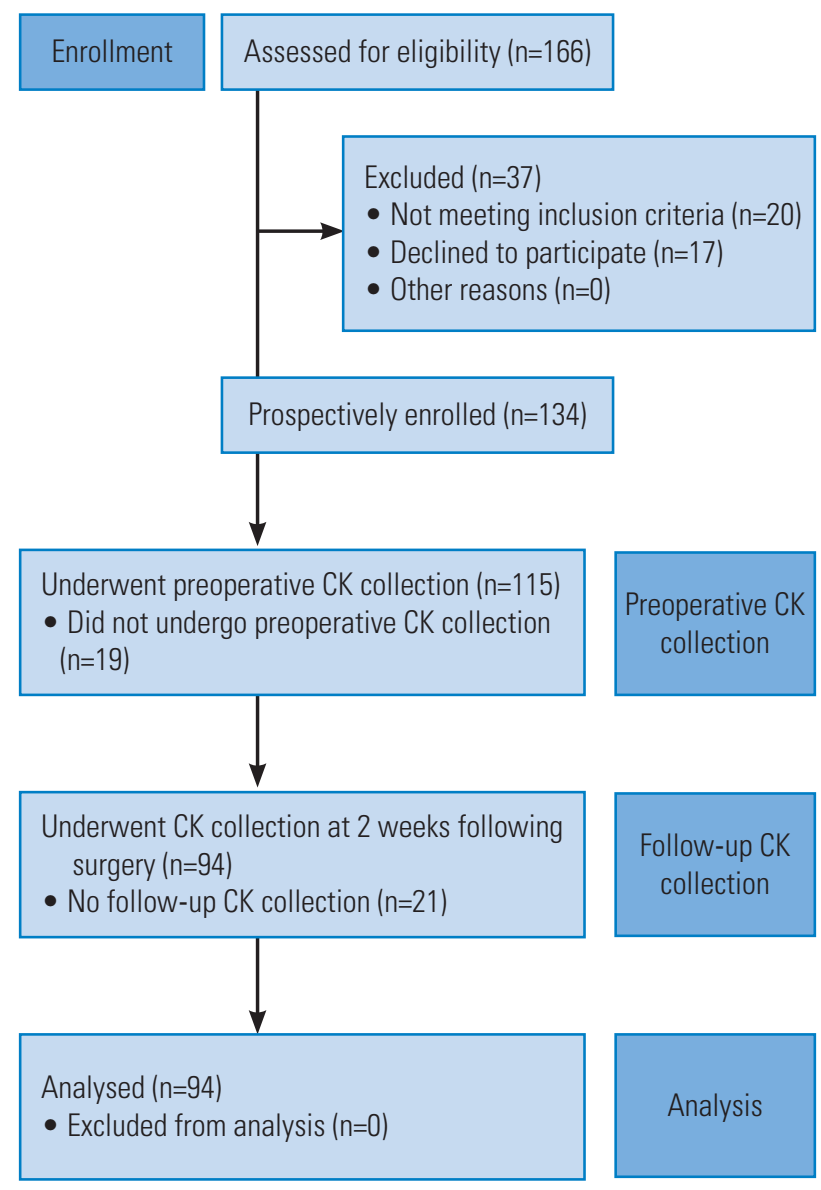

Fig. 1. Consort flow diagram for patients. CK, creatine kinase. 
Table 2. Procedure performed and number of patients undergoing each procedure

\begin{tabular}{|c|c|}
\hline Procedure & No. of patients \\
\hline Posterior cervical & 3 \\
\hline C1-C2 posterior spinal fusion & 1 \\
\hline C3-C6 posterior decompression and fusion & 1 \\
\hline C3-T1 posterior decompression and fusion & 1 \\
\hline Anterior cervical & 35 \\
\hline Corpectomy & 7 \\
\hline ACDF & 16 \\
\hline Single level & 11 \\
\hline 2 Level & 3 \\
\hline 3 Level & 2 \\
\hline Total disc replacement & 10 \\
\hline Hybrid ACDF/TDR & 2 \\
\hline Anterior/posterior & 1 \\
\hline $\begin{array}{l}\text { C6 corpectomy, C4-C5 ACDF, anterior plating } \\
\text { C4-C7, posterior instrumented fusion C4-C7 }\end{array}$ & 1 \\
\hline Anterior lumbar & 13 \\
\hline L3-L4 XLIF & 1 \\
\hline L5-S1 TDR & 1 \\
\hline L5-S1 ALIF & 10 \\
\hline L4-L5, L5-S1 TDR & 1 \\
\hline L4-L5 TDR, L5-S1 ALIF & 1 \\
\hline Posterior lumbar & 38 \\
\hline Discectomy & 12 \\
\hline Posterior fusion & 12 \\
\hline Posterior interbody fusion & 11 \\
\hline Combined posterior/anterior lumbar & 2 \\
\hline
\end{tabular}

ACDF, anterior cervical discectomy and fusion; TDR, total disc replacement; XLIF, extreme lateral interbody fusion; ALIF, anterior lumbar interbody fusion.

and prospective evaluation (70\% male, 30\% female; average age, $47.58 \pm 10.77$ years) (Table 1, Fig. 1). None of 94 patients received a postoperative diagnosis of rhabdomyolysis.

Surgical procedures are summarized in Table 2. A total 57 patients underwent lumbar surgery, and 37 underwent cervical spine surgery, with 41 procedures being performed in the prone position and 48 in the supine position. A majority of the patients (52\%) were white, $32 \%$ were black, and $10 \%$ were from other ethnic groups. The patients were divided into three groups based on the number of levels operated on; the three groups were one level $(n=61)$, two levels $(n=21)$, and three or more levels $(\mathrm{n}=12)$. Instrumentation was utilized for $85 \%$ of the patients, and only six patients underwent revision surgery, while 88 underwent primary surgery.

The surgical variables and the average $\mathrm{CK}$ values are presented in Table 3. The preoperative CK, CK at POD 1, CK change (difference) at POD 1 , and CK change (ratio) at POD 1 were examined. The preoperative $\mathrm{CK}$ values ranged from 29-779 U/L. Overall, 45 of the 94 patients (48\%) had an elevated preoperative CK value, defined as a value $>150$ U/L. Preoperative CK values were not significantly correlated with the CK values at POD 1, CK change (difference) at POD 1, or CK change (ratio) at POD 1.

The preoperative $\mathrm{CK}$ levels were significantly higher for men than for women $(p=0.0082)$. Despite higher baseline CK levels in men, there were no significant sex-based differences in the CK values at POD $1(p=0.2846)$, CK change (difference) to POD 1 ( $p=0.8739$ ), and CK change (ratio) to POD 1 ( $p=0.2525)$. The variability was proportionally higher for men (Table 4 ).

When the CK variables were assessed based on race, a trend for the magnitude of the CK variables between the races was observed, though not statistically significant. African-American patients had the highest preoperative CK values ( $p=0.0824)$ and POD 1 CK values $(p=0.2076)$; furthermore, this ethnic group showed the greatest change in both difference $(p=0.3968)$ and ratio $(p=0.3419)$ (Table 5).

Comparing the change in the CK level at POD 1 between the cervical and lumbar cases, the mean difference in the CK change between the preoperative and POD $1 \mathrm{CK}$ values was $450.08 \mathrm{U} / \mathrm{L}$ for the cervical group and $811.25 \mathrm{U} / \mathrm{L}$ for the lumbar group; however, this difference was not significantly significant $(p=0.052)$. However, the ratio of the $\mathrm{CK}$ change from preoperative to that at $\mathrm{POD}$ 1 was 3.65 in the cervical group and 6.05 in the lumbar group, representing a significant difference $(p=0.014)$ (Table 6).

Looking at the difference in the preoperative and POD $1 \mathrm{CK}$ values in the prone and supine positions, the prone position patients had a mean difference of 884.90 as compared to the supine position patients who had a mean difference of $478.85 \mathrm{U} / \mathrm{L}$. Both the differences and ratios of the change were statistically significant ( $p=0.022$ and $p=0.0055$, respectively) (Table 7). Within the subgroups, defined by patient position and spinal region operated on, the CK change (difference) to POD 1 was not signifi- 
Table 3. Surgical variables and average CK levels

\begin{tabular}{|c|c|c|c|c|c|}
\hline Variable & No. of patients & Mean \pm standard deviation & Median & Min & $\operatorname{Max}$ \\
\hline Age (yr) & 94 & $47.55 \pm 10.79$ & 46.5 & 24 & 69 \\
\hline Body mass index $\left(\mathrm{kg} / \mathrm{m}^{2}\right)$ & 94 & $28.98 \pm 4.14$ & 28.4 & 17.53 & 42.45 \\
\hline Blood loss (mL) & 94 & $239.43 \pm 417.83$ & 100 & 10 & 2,500 \\
\hline Intraoperative fluids (mL) & 90 & $1,411.11 \pm 685.15$ & 1,250 & 100 & 4,000 \\
\hline Length of surgery (min) & 94 & $299.13 \pm 168.89$ & 290 & 101 & 992 \\
\hline Preoperative creatinine (mg/dL) & 94 & $1.00 \pm 0.80$ & 0.9 & 0.5 & 8.4 \\
\hline Preoperative CK (U/L) & 94 & $179.64 \pm 139.50$ & 140.5 & 29 & 779 \\
\hline POD 1 CK (U/L) & 93 & $847.04 \pm 1,246.21$ & 490 & 100 & 8,828 \\
\hline POD 2 CK (U/L) & 53 & $907.58 \pm 1,154.67$ & 525 & 108 & 7,220 \\
\hline POD 3 CK (U/L) & 13 & $872.69 \pm 1,117.90$ & 374 & 82 & 3,447 \\
\hline POD 14 CK (U/L) & 89 & $118.56 \pm 91.41$ & 101 & 38 & 756 \\
\hline CK change (difference) at POD 1 (U/L) & 93 & $667.56 \pm 1,241.63$ & 291 & -180 & 8,724 \\
\hline CK change (ratio) at POD $1^{*}(\mathrm{U} / \mathrm{L})$ & 93 & $5.10 \pm 10.43$ & 2.6 & -0.23 & 83.88 \\
\hline
\end{tabular}

CK, creatine kinase; POD, postoperative day. * $p<0.05$.

Table 4. CK level as a function of patient sex

\begin{tabular}{|c|c|c|c|c|c|c|c|c|}
\hline Outcome & Sex & No. of patients & Mean \pm standard deviation & Median & Min & Max & Statistic & $p$-value ${ }^{\text {a) }}$ \\
\hline \multirow[t]{2}{*}{ Preoperative CK } & M & 66 & $194.70 \pm 138.28$ & 154.5 & 53 & 779 & 1,003 & 0.0082 \\
\hline & $\mathrm{F}$ & 28 & $144.14 \pm 138.32$ & 98.5 & 29 & 750 & & \\
\hline \multirow[t]{2}{*}{ CK at POD 1} & M & 65 & $967.45 \pm 1453.86$ & 505 & 165 & 8,828 & 1,187 & 0.2846 \\
\hline & $\mathrm{F}$ & 28 & $567.54 \pm 407.03$ & 473 & 100 & 1,621 & & \\
\hline \multirow[t]{2}{*}{ CK change (difference) to POD 1} & M & 65 & $772.74 \pm 1453.22$ & 291 & -180 & 8,724 & $1,296.5$ & 0.8739 \\
\hline & $\mathrm{F}$ & 28 & $423.39 \pm 398.34$ & 290 & -110 & 1,478 & & \\
\hline \multirow[t]{2}{*}{ CK change (ratio) to POD 1} & M & 65 & $5.45 \pm 12.23$ & 2.00 & -0.23 & 83.9 & 1,454 & 0.2525 \\
\hline & $\mathrm{F}$ & 28 & $4.28 \pm 3.92$ & 3.69 & -0.15 & 17.4 & & \\
\hline
\end{tabular}

$\mathrm{M}$, male; F, female; CK, creatine kinase; POD, postoperative day.

a) By Wilcoxon rank-sum test.

cantly different among the four groups: prone and cervical, supine and cervical, prone and lumbar, and supine and lumbar $(p=0.109)$. However, the CK change (ratio) to POD 1 was significantly different among the four groups $(p=0.026)$. Using Dunn's post-hoc multiple comparison test to compare the medians, the supine and cervical group was significantly different from the prone and lumbar group $(p<0.05)$ (Table 8$)$.

There was no significant difference in the CK change (difference) to POD 1 or CK change (ratio) to POD 1 among the patients with one, two, or three or more levels operated on $(p=0.210$ and $p=0.379)$ (Table 9). The CK change (difference) to POD 1 values was significantly higher for instrumentation versus no instrumentation $(p=0.017)$. There was no significant difference in the CK change (ratio) to POD 1 in the two groups ( $p=0.123$ ); this was also calculated using Wilcoxon rank-sum test (Table 10). Despite only six revision cases, the CK change (difference) to POD $1(p=0.0035)$ and CK change (ratio) to POD 1 ( $p=0.007)$ values were significantly higher for the revision versus no revision cases, as calculated using Wilcoxon rank-sum test (Table 11).

\section{Discussion}

In this prospective study, we failed to identify any cases of 
Table 5. Comparison of CK level as a function of patient ethnicity

\begin{tabular}{|c|c|c|c|c|c|c|c|c|}
\hline Variable & Race & $\begin{array}{c}\text { No. of } \\
\text { patients }\end{array}$ & $\begin{array}{c}\text { Mean } \pm \text { standard } \\
\text { deviation }\end{array}$ & Median & Min & $\operatorname{Max}$ & Statistic & $p$-value ${ }^{\text {a) }}$ \\
\hline \multirow[t]{3}{*}{ Preoperative CK } & Black & 32 & $216.78 \pm 160.63$ & 172.5 & 29 & 779 & 4.99 & 0.0824 \\
\hline & White & 52 & $160.58 \pm 133.66$ & 118.5 & 49 & 750 & & \\
\hline & Other & 10 & $159.90 \pm 59.47$ & 142.5 & 87 & 285 & & \\
\hline \multirow[t]{3}{*}{ CK at POD 1} & Black & 32 & $1,204.97 \pm 1,727.35$ & 567 & 196 & 8,828 & 3.14 & 0.2076 \\
\hline & White & 51 & $702.24 \pm 928.09$ & 490 & 100 & 6,514 & & \\
\hline & Other & 10 & $440.20 \pm 233.87$ & 348.5 & 233 & 929 & & \\
\hline \multirow[t]{3}{*}{ CK change (difference) to POD 1} & Black & 32 & $988.19 \pm 1,725.40$ & 373 & -180 & 8,724 & 1.85 & 0.3968 \\
\hline & White & 51 & $542.31 \pm 932.54$ & 286 & -110 & 6,392 & & \\
\hline & Other & 10 & $280.30 \pm 229.01$ & 227.5 & 21 & 776 & & \\
\hline \multirow[t]{3}{*}{ CK change (ratio) to POD 1} & Black & 32 & $6.73 \pm 14.84$ & 2.00 & -0.23 & 83.88 & 2.15 & 0.3419 \\
\hline & White & 51 & $4.68 \pm 7.71$ & 2.97 & -0.16 & 52.39 & & \\
\hline & Other & 10 & $1.98 \pm 1.65$ & 1.59 & 0.09 & 5.07 & & \\
\hline
\end{tabular}

CK, creatine kinase; POD, postoperative day.

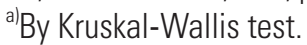

Table 6. Comparison of the change in the CK level at POD 1 (cervical vs. lumbar)

\begin{tabular}{|c|c|c|c|c|c|c|c|c|}
\hline Variable & Group & $\begin{array}{c}\text { No. of } \\
\text { patients }\end{array}$ & $\begin{array}{c}\text { Mean } \pm \text { standard } \\
\text { deviation }\end{array}$ & Median & Min & Max & Statistic & $p$-value ${ }^{\mathrm{a})}$ \\
\hline \multirow[t]{2}{*}{ CK change (difference) to POD 1} & Cervical & 37 & $450.08 \pm 1,028.44$ & 260.00 & -180 & 6,392 & 1,488 & 0.052 \\
\hline & Lumbar & 56 & $811.25 \pm 1,354.10$ & 344.50 & -110 & 8,724 & & \\
\hline \multirow[t]{2}{*}{ CK change (ratio) to POD 1} & Cervical & 37 & $3.65 \pm 8.60$ & 1.77 & -0.23 & 52.39 & 1,418 & 0.014 \\
\hline & Lumbar & 56 & $6.05 \pm 11.46$ & 3.63 & -0.15 & 83.88 & & \\
\hline
\end{tabular}

CK, creatine kinase; POD, postoperative day.

a) By Wilcoxon rank-sum test.

Table 7. Comparison of the change in the CK level at POD 1 (prone vs. supine)

\begin{tabular}{llccccccc} 
Variable & Group & $\begin{array}{c}\text { No. of } \\
\text { patients }\end{array}$ & $\begin{array}{c}\text { Mean } \pm \text { standard } \\
\text { deviation }\end{array}$ & Median & Min & Max & Statistic $p$-value \\
CK change (difference) to POD 1 & Prone & 41 & $884.90 \pm 1,532.41$ & 382.00 & -110 & 8,724 & $2,128.5$ & 0.022 \\
& Supine & 48 & $478.85 \pm 960.08$ & 248.50 & -180 & 6,392 & \\
CK change (ratio) to POD 1 & Prone & 41 & $6.90 \pm 13.18$ & 3.87 & -0.15 & 83.88 & 2,191 & 0.0055 \\
& Supine & 48 & $3.29 \pm 7.54$ & 1.78 & -0.23 & 52.39 & \\
\hline
\end{tabular}

CK, creatine kinase; POD, postoperative day.

a) By Wilcoxon rank-sum test.

rhabdomyolysis among patients undergoing cervical or lumbar spine surgery. The average change in the CK levels from the preoperative value to that at POD 1 for lumbar spine surgery was $811.25 \mathrm{U} / \mathrm{L}$, and the average change for cervical surgery cases was $450.08 \mathrm{U} / \mathrm{L}$. The change ratio was 6.05 and 3.65 for lumbar and cervical surgery, respec- tively. In a similar manner, the average change was 884.90 $\mathrm{U} / \mathrm{L}$ and $478.85 \mathrm{U} / \mathrm{L}$, with change ratios of 6.90 and 3.29 for prone and supine positioning, respectively. Furthermore, the largest change was seen with prone lumbar surgery, while the lowest change was seen for supine cervical surgery. Finally, the revision procedures had statistically 
Table 8. Comparison of the change in the CK level at POD 1 (among the four groups defined by the position and spinal region)

\begin{tabular}{|c|c|c|c|c|c|c|c|c|c|}
\hline Variable & Group & $\begin{array}{c}\text { No. of } \\
\text { patients }\end{array}$ & $\begin{array}{c}\text { Mean } \pm \text { standard } \\
\text { deviation }\end{array}$ & Median & Min & Max & $X^{2}$ & $\begin{array}{l}\text { Degrees of } \\
\text { freedom }\end{array}$ & $p$-value \\
\hline \multirow[t]{4}{*}{ CK change (difference) to POD 1} & Supine/cervical & 33 & $440.27 \pm 1,087.58$ & 250.00 & -180 & 6,392 & 6.06 & 3 & 0.109 \\
\hline & Supine/lumbar & 15 & $563.73 \pm 616.16$ & 247.00 & 25 & 1,825 & & & \\
\hline & Prone/cervical & 3 & $471.33 \pm 277.35$ & 558.00 & 161 & 695 & & & \\
\hline & Prone/lumbar & 38 & $917.55 \pm 1,587.31$ & 374.50 & -110 & 8,724 & & & \\
\hline \multirow[t]{4}{*}{ CK change (ratio) to POD 1} & Supine/cervical & 33 & $3.48 \pm 8.94$ & 1.76 & -0.23 & 52.39 & 9.24 & 3 & 0.026 \\
\hline & Supine/lumbar & 15 & $2.89 \pm 2.85$ & 1.97 & 0.12 & 11.34 & & & \\
\hline & Prone/cervical & 3 & $2.32 \pm 1.49$ & 1.96 & 1.03 & 3.95 & & & \\
\hline & Prone/lumbar & 38 & $7.26 \pm 13.63$ & 4.13 & -0.15 & 83.88 & & & \\
\hline
\end{tabular}

CK, creatine kinase; POD, postoperative day.

a) By Kruskal-Wallis test.

Table 9. Comparison of the change in the CK level relative to the number of surgical levels

\begin{tabular}{|c|c|c|c|c|c|c|c|c|c|}
\hline Variable & $\begin{array}{l}\text { No. of } \\
\text { levels }\end{array}$ & $\begin{array}{c}\text { No. of } \\
\text { patients }\end{array}$ & $\begin{array}{c}\text { Mean } \pm \text { standard } \\
\text { deviation }\end{array}$ & Median & Min & Max & $x^{2}$ & $\begin{array}{l}\text { Degrees of } \\
\text { freedom }\end{array}$ & $p$-value ${ }^{a)}$ \\
\hline \multirow[t]{3}{*}{ CK change (difference) to POD 1} & 1 & 60 & $558.18 \pm 984.19$ & 250.50 & -31 & 6,392 & 3.12 & 2 & 0.210 \\
\hline & 2 & 21 & $719.05 \pm 941.56$ & 367.00 & -180 & 3,897 & & & \\
\hline & $\geq 3$ & 12 & $1,124.33 \pm 2,405.93$ & 425.50 & 111 & 8,724 & & & \\
\hline \multirow[t]{3}{*}{ CK change (ratio) to POD 1} & 1 & 60 & $4.10 \pm 7.28$ & 2.08 & -0.16 & 52.39 & 1.94 & 2 & 0.379 \\
\hline & 2 & 21 & $4.80 \pm 4.85$ & 2.75 & -0.23 & 16.89 & & & \\
\hline & $\geq 3$ & 12 & $10.56 \pm 23.36$ & 3.31 & 0.99 & 83.88 & & & \\
\hline
\end{tabular}

CK, creatine kinase; POD, postoperative day.

a)By Wilcoxon rank-sum test.

Table 10. Comparison of the change in the CK level at POD 1 for instrumentation (yes/no)

\begin{tabular}{lcccccccc} 
Variable & Instrumentation & $\begin{array}{c}\text { No. of } \\
\text { patients }\end{array}$ & $\begin{array}{c}\text { Mean } \pm \text { standard } \\
\text { deviation }\end{array}$ & Median & Min & Max & Statistic $p$-value \\
CK change (difference) to POD 1 & Yes & 80 & $743.65 \pm 1,323.01$ & 332 & -180 & 8,724 & 391 & 0.017 \\
& No & 13 & $199.31 \pm 140.91$ & 160 & -31 & 506 & \\
\hline CK change (ratio) to POD 1 & Yes & 80 & $5.46 \pm 11.07$ & 2.84 & -0.23 & 83.88 & 470 & 0.123 \\
& No & 13 & $2.87 \pm 4.59$ & 1.44 & -0.16 & 17.45 & \\
\hline
\end{tabular}

CK, creatine kinase; POD, postoperative day.

a) By Wilcoxon rank-sum test.

Table 11. Comparison of the change in the CK level at POD 1 for revision (yes/no)

\begin{tabular}{lcccccccc} 
Variable & Revision & $\begin{array}{c}\text { No. of } \\
\text { patients }\end{array}$ & $\begin{array}{c}\text { Mean } \pm \text { standard } \\
\text { deviation }\end{array}$ & Median & Min & Max & Statistic $p$-value ${ }^{\text {a) }}$ \\
CK change (difference) to POD 1 & Yes & 6 & $2,079.17 \pm 3,290.83$ & 662.50 & 208 & 8,724 & 419 & 0.035 \\
& No & 87 & $570.21 \pm 933.25$ & 278.00 & -180 & 6,392 & \\
CK change (ratio) to POD 1 & Yes & 6 & $19.90 \pm 31.54$ & 6.57 & 2.93 & 83.88 & 460 & 0.007 \\
& No & 87 & $4.07 \pm 6.50$ & 2.00 & -0.23 & 52.39 & \\
\hline
\end{tabular}

CK, creatine kinase; POD, postoperative day.

a) By Wilcoxon rank-sum test. 
significant increases in the CK levels and the CK ratios, while the instrumentation produced statistically significant increases in the change in the CK levels alone. There was no significant difference with respect to the body mass index, age, sex, or ethnicity.

These results are similar to those reported by Kawaguchi et al. [5] in 1997. They looked at the CK-MB isoenzyme rise following both anterior and posterior lumbar procedures to find that the CK levels rose more for posterior approaches than for the anterior approaches, and multilevel surgery showed higher rises than single level surgery [5]. This study, however, did not include any patients who underwent posterior instrumentation [5]. Further, in 2007, Arts et al. [6] showed that maximally invasive surgery led to a statistically significant increase in the preoperative/postoperative CK ratio compared to average and minimally invasive surgery. In a similar manner, there was a lower change when cervical surgery was compared to thoracic and lumbar surgery and to anterior to posterior procedures, although the changes that they saw were smaller than those in the current study [6]. Finally, in 1997, Lenke et al. [7] examined the increase in CK-MB isoenzyme following spine surgery. They found that patients with more than one operative level showed increases in the enzyme level above the upper limit of normal, and a larger number of involved levels gave a higher rise in the CK-MB. This study, however, grouped all the patients together, for a multitude of different diagnoses and procedures. There was no division between the spinal levels or the approaches [7].

Clearly, the results of this study are comparable to those of other studies, showing the expected rises in the CK levels after various types of spine surgery; this may help define the normative values for postoperative $\mathrm{CK}$ and in cases where a rise may be concerning. The results may be confounded by the muscular damaged produced by the dissection and retraction of the paraspinal musculature in prone/lumbar surgery; however, one cannot minimize the effect of positioning on muscular damage. During the prone procedures at our institution, the patients are positioned with a chest pad, pads on the iliac crest, and additional pads on the anterior compartment of the thigh. Pillows are placed under the legs. In contrast, the patients undergoing supine procedures are typically positioned on flat top tables with their weight distributed evenly through the scapula, spine, buttock, posterior thighs, and legs. The differences in the positioning obviously place more pres- sure on the anterior compartment of the thighs during prone positioning that could contribute to the postoperative CK rises. Moreover, posterior approaches are typically lengthier; revision cases and cases with instrumentation further lengthen the surgical time, all of which can increase the pressure on muscular compartments from positioning. This result is similar to the observations made in previous reports. Keene et al. [8] reported the case of a patient with bilateral gluteal compartment syndrome following prolonged positioning for a prostatectomy, Cascio et al. [9] described two cases of compartment syndrome due to prolonged lateral positioning, and Seybold and Busconi [10] described a case of anterior thigh compartment syndrome caused by prolonged lateral positioning during a scapular fasciocutaneous free flap. In comparison, prone/ posterior and supine/anterior cervical surgery cause very similar changes in the CK levels, with the prone/posterior group showing a non-statistically significant elevation in the CK levels. However, it is challenging to draw conclusions from this group because the number of posterior cervical patients was very low in this study.

The present results should be viewed in light of the study limitations. First, of the initial 134 patients included in the study, forty could not be analyzed due to incomplete collection of data at their preoperative appointment or during the follow-up period. Moreover, the positioning and approach data included patients undergoing surgery both with and without instrumentation and at multiple levels; thus, this may not reflect the true magnitude of the rise in the CK levels when extrapolating to only patients undergoing prone/lumbar surgery for decompression versus those also undergoing fusion procedures. In a similar manner, the large rise in the CK levels observed in prone positioning may be artificially increased owing to the dissection for posterior lumbar surgery in addition to that because of the muscular injury from positioning. Further, while there was a statistically significant change in the CK levels in patients undergoing revision surgery, only six patients underwent revision surgery during the study period. Finally, as mentioned earlier, this is a very heterogeneous sample with a relatively low samples size in some of the groups (prone/cervical); this may also inhibit our ability to extrapolate to different patient subsets.

Thus, the current study refers to normal rises in the CK levels following various types of spine surgery. Similar to previous reports, the present trial shows that the largest risk for a rise in the CK level is with prone/lumbar proce- 
dures that are lengthier and require access to more levels. This rise in the CK levels may represent injury to the paraspinal musculature, which, if extensive, may lead to rhabdomyolysis. Thus, the surgeon should minimize the risk of muscle damage during long, posterior lumbar surgeries by releasing the retractors regularly and decreasing direct muscle damage during dissection to minimize the risk of postoperative rhabdomyolysis.

\section{Conclusions}

In conclusion, this study showed that the CK levels elevated following spine surgery; this rise was most notable in the prone, lumbar group. While some of the elevation may be attributable to paraspinal muscle damage, the damage to the muscular compartments due to increased pressure in the prone position also contributed to this change. This study can be used to help determine an expected rise in the CK levels following spine surgery and shows that unless there is an exceptionally large amount of muscle damage, the $\mathrm{CK}$ rise does not approach dangerous levels.

\section{Conflict of Interest}

No potential conflict of interest relevant to this article was reported. Each author certifies that he or she has no commercial associations that might pose a conflict of interest in connection with the submitted article. No funding was received for this study.

\section{References}

1. Weiss AJ, Elixhauser A. Trends in operating room procedures in U.S. hospitals, 2001-2011 [Internet]. Rockville (MD): Healthcare Cost and Utilization
Project; 2014 [cited 2018 Jul 10]. Available from: https://www.hcup-us.ahrq.gov/reports/statbriefs/ sb171-Operating-Room-Procedure-Trends.jsp.

2. Holt SG, Moore KP. Pathogenesis and treatment of renal dysfunction in rhabdomyolysis. Intensive Care Med 2001;27:803-11.

3. Dakwar E, Rifkin SI, Volcan IJ, Goodrich JA, Uribe JS. Rhabdomyolysis and acute renal failure following minimally invasive spine surgery: report of 5 cases. J Neurosurg Spine 2011;14:785-8.

4. Foster MR. Rhabdomyolysis in lumbar spine surgery: a case report. Spine (Phila Pa 1976) 2003;28:E276-8.

5. Kawaguchi Y, Matsui H, Tsuji H. Changes in serum creatine phosphokinase MM isoenzyme after lumbar spine surgery. Spine (Phila Pa 1976) 1997;22:1018-23.

6. Arts MP, Nieborg A, Brand R, Peul WC. Serum creatine phosphokinase as an indicator of muscle injury after various spinal and nonspinal surgical procedures. J Neurosurg Spine 2007;7:282-6.

7. Lenke LG, Bridwell KH, Jaffe AS. Increase in creatine kinase MB isoenzyme levels after spinal surgery. J Spinal Disord 1994;7:70-6.

8. Keene R, Froelich JM, Milbrandt JC, Idusuyi OB. Bilateral gluteal compartment syndrome following robotic-assisted prostatectomy. Orthopedics 2010;33:852.

9. Cascio BM, Buchowski JM, Frassica FJ. Well-limb compartment syndrome after prolonged lateral decubitus positioning: a report of two cases. J Bone Joint Surg Am 2004;86-A:2038-40.

10. Seybold EA, Busconi BD. Anterior thigh compartment syndrome following prolonged tourniquet application and lateral positioning. Am J Orthop (Belle Mead NJ) 1996;25:493-6. 